\title{
Efficacy of Statin Therapy in Patients with Hospital Admission for COVID-19
}

\author{
Daein Choi ${ }^{1}$ Q Qinzhong Chen ${ }^{2}$ Sascha N. Goonewardena ${ }^{3} \cdot$ Hannah Pacheco $^{2}$ - Priscilla Mejia ${ }^{2}$ Robin L. Smith ${ }^{4}$ \\ Robert S. Rosenson ${ }^{2}$
}

Accepted: 7 September 2021 / Published online: 15 September 2021

(c) Springer Science+Business Media, LLC, part of Springer Nature 2021

\begin{abstract}
Purpose COVID-19 is characterized by dysfunctional immune responses and metabolic derangements, which in some, lead to multi-organ failure and death. Statins are foundational lipid-lowering therapeutics for cardiovascular disease and also possess beneficial immune-modulating properties. Because of these immune-modulating properties, some have suggested their use in COVID-19. We sought to investigate the association between statin use and mortality in patients hospitalized with COVID-19.

Methods Five thousand three hundred seventy-five COVID-19 patients admitted to Mount Sinai Health System hospitals in New York between February 27, 2020, and December 3, 2020, were included in this analysis. Statin use was classified as either non-user, low-to-moderate-intensity user, or high-intensity user. Multivariate Cox proportional hazards models were used to evaluate in-hospital mortality rate. Considered covariates were age, sex, race, and comorbidities.

Results Compared to non-statin users, both low-to-moderate-intensity (adjusted hazard ratio; aHR 0.62, 95\% confidential intervals; CI 0.51-0.76) and high-intensity statin users (aHR 0.53, 95\% CI 0.43-0.65) had a reduced risk of death. Subgroup analysis of 723 coronary artery disease patients showed decreased mortality among high-intensity statin users compared to non-users (aHR 0.51, 95\% CI 0.36-0.71).

Conclusions Statin use in patients hospitalized with COVID-19 was associated with a reduced in-hospital mortality. The protective effect of statin was greater in those with coronary artery disease. These data support continued use of statin therapy in hospitalized patients with COVID-19. Clinical trials are needed to prospectively determine if statin use is effective in lowering the mortality in COVID-19 and other viral infections.
\end{abstract}

Keywords Statin $\cdot$ COVID-19 $\cdot$ Mortality $\cdot$ Coronary artery disease

Robert S. Rosenson

robert.rosenson@mssm.edu

1 Department of Medicine, Mount Sinai Beth Israel, Icahn School of Medicine at Mount Sinai, New York, NY, USA

2 Metabolism and Lipids Unit, Cardiovascular Institute, Marie-Josee and Henry R Kravis Center for Cardiovascular Health, Icahn School of Medicine at Mount Sinai, The Mount Sinai Medical Center, One Gustave L. Levy Place, Box 1030, New York, NY 10029, USA

3 Division of Cardiovascular Medicine, Department of Internal Medicine, University of Michigan, Ann Arbor, MI, USA

4 Clinical Associate Professor, Department of Medicine, Rutgers New Jersey Medical School and the CURA Foundation, New York, NY, USA

\section{Introduction}

The severe acute respiratory syndrome coronavirus 2 (SARS-CoV-2) has affected 224 million people and resulted in 4.6 million deaths worldwide as of 13 September 2021 [1], becoming an unprecedented pandemic in modern history. Coronavirus disease-2019 (COVID-19), the disease caused by SARS-CoV-2, incites a systemic inflammatory response leading to tissue injury and organ failure [2]. Multiple mRNA vaccines and immunotherapies have been developed to prevent symptomatic infections of SARS-CoV-2. However, questions remain on the durability of immunity and efficacy against viral variants and there is still no known definitive treatment for COVID-19. Therefore, it remains critical to understand the mechanisms that drive 
the pathogenicity of COVID-19 and the patients most at risk of COVID-19 complications.

COVID-19 may cause severe complications including myocardial injury, thromboembolic disease, and acute respiratory distress syndrome [3]. Higher rates of cardiovascular morbidity and mortality in COVID-19 patients have been reported in those with comorbidities including older age, hypertension, diabetes, chronic kidney disease, atherosclerotic cardiovascular disease, and chronic heart failure $[4,5]$. Thus, many of these patients with cardiac injury have baseline clinical characteristics that identify them as very high risk for cardiovascular events [6]. Viral illnesses including COVID-19 incite a profound systemic inflammatory response leading to tissue injury and organ failure. Among patients with COVID-19, cardiac injury is associated with higher leukocyte counts and elevated concentrations of procalcitonin and C-reactive protein [5].

Statin therapy is the first-line treatment for lowering cholesterol in patients at high risk for cardiovascular events. These agents target low-density lipoprotein (LDL), but they possess potent anti-inflammatory, immune-modulating and anti-thrombic properties that might be effective in an acute setting of COVID-19 [7-10]. On the other hand, statins are also known to increase the expression of angiotensin-converting enzyme 2 (ACE2) [11-13], which is a membranebound aminopeptidase in tissues that acts as a receptor of SARS-CoV-2 viral entry [11, 14]. In addition, statins are associated with adverse muscle events that may be accentuated in patients with systemic inflammation and impaired drug elimination pathways with acute organ failure [15]. These considerations have raised concerns regarding the safety and efficacy of statins in patients with acute COVID19 infections.

Few previous studies have investigated the effect of statin on COVID-19. Earlier studies on statin use prior to hospital admission reported an association with decreased mortality and severity of the disease [16-19]; however, the effectiveness of statin therapy on cardiovascular morbidity and mortality in hospitalized COVID-19 patients is still controversial. While some observational studies have reported that in-hospital uses of statins were associated with favorable clinical outcomes among COVID-19 patients [20-22], the findings were limited by the lack of potential confounders, lack of laboratory data, low sample size, and lack of ability to assess the dose of statins. Since some other studies have failed to demonstrate the protective effect of statin [ 16 , $23,24]$, there is no consensus of statin use on hospitalized COVID-19 patients up to now.

As statins are commonly used medications especially among high-risk subgroups who become infected with SARS-CoV2, urgent research is needed to investigate the safety and efficacy of statin therapy in patients hospitalized with COVID-19. Thus, we sought to evaluate the effect of statin therapy in hospitalized COVID-19 patients among a large cohort of hospitalized patients in New York City.

\section{Methods}

\section{Data Collection}

Data collection was conducted from electronic health records from the 6 hospitals of the Mount Sinai Health System (MSHS). Demographic information, comorbidities, vital signs and laboratory measurements during hospitalization, dispensed medications, in-hospital oxygen requirements, and outcomes (death or hospital discharge) were collected. Collected demographics included age, sex, race, and ethnicities.

\section{Study Population}

The study population was derived from six New York City hospitals of MSHS: Mount Sinai Beth Israel, Mount Sinai Brooklyn, Mount Sinai Hospital, Mount Sinai Morningside, Mount Sinai Queens, and Mount Sinai West. We included a total of 5375 laboratory-confirmed COVID-19 patients who were admitted to MSHS hospitals and diagnosed with COVID-19 within $48 \mathrm{~h}$ of presentation from February 27, 2020, to December 3, 2020. The confirmation of COVID19 infection was defined by positive reverse transcriptase polymerase chain reaction assay of a specimen collected by nasopharyngeal swab. Participants were classified into non-statin users, low-to-moderate-intensity statin users, and high-intensity statin users, based on statin prescription after COVID-19 diagnosis. High-intensity statin was defined by the use of atorvastatin $40 \mathrm{mg}$ or higher daily or rosuvastatin $20 \mathrm{mg}$ or higher daily. Analysis including laboratory profiles was performed on a subgroup of 3411 participants who had a measurement of inflammatory markers within $24 \mathrm{~h}$ of confirmation of COVID-19 infection.

\section{Statistical Analysis}

Continuous variables are reported as means and standard deviations. Categorical variables were summarized as counts and percentages. Statistical significances were evaluated by analysis of variance method for continuous variables and chi-square test for categorical variables. Patients who were discharged alive from the hospital, or still hospitalized at the time of data freeze, were regarded as right-censored. Multivariate Cox proportional hazards models were used to evaluate the in-hospital mortality rate. Considered covariates were age, sex, race, oxygen support device on presentation, comorbidities of hypertension, diabetes mellitus, atrial fibrillation, congestive heart failure, chronic kidney disease, and coronary artery disease (CAD). Kaplan-Meier 
curve for survival rate was plotted by statin use (Fig. 1). Additional multivariate analysis including initial vital signs and initial laboratory data including inflammatory markers (white blood cell count, C-reactive protein, and D-dimer) was conducted among participants with laboratory profiles. Stratified analysis according to the subgroups of age, sex, race, baseline comorbidities (hypertension, diabetes mellitus, atrial fibrillation, congestive heart failure, chronic kidney disease, and CAD), hospital course (intensive care unit admission, intubation), and baseline laboratory profiles (white blood cell count, C-reactive protein level, D-dimer level) was conducted (Fig. 2). Additionally, a sensitivity analysis of in-hospital statin compliance was performed. Incomplete statin therapy was defined by medication possession ratio (MPR) lower than 0.8 during the hospital stay [25-27]. Supplementary analysis was conducted to assess the in-hospital mortality of incomplete statin users compared to statin non-users. Lastly, propensity score matching was conducted using the greedy method with a caliper of 0.1 times the standard deviation of logit propensity score. All covariates were taken into account for the 1:1 matching of statin users to non-statin using controls. Lastly, we conducted falsification endpoint analysis by having endpoint of severe anemia requiring blood transfusion among COVID19 patients, which is not expected to be influenced by statin use [28, 29]. All statistical analyses were performed with SAS Enterprise Guide 8.1 (SAS Institute Inc., Cary, NC).

\section{Ethical Consideration}

The Mount Sinai Institutional Review Board approved this study (IRB number: $20-03,558$ ). The individual data were anonymized and strictly deidentified prior to distribution to researchers and the requirement for informed consent was waived.

\section{Results}

Descriptive characteristics of the study population are depicted in Table 1. Among 5375 COVID patients hospitalized during the study period, $877(16.3 \%)$ were treated with low-to-moderate-intensity statin and $843(15.7 \%)$ were treated with the high-intensity statin. The average age of non-statin users was 60.2 (standard deviation, SD 18.9) years, while statin users were older with an average of 71.5 (SD 12.2) years among low-to-moderate-intensity statin users and 69.7 (12.7) years on high-intensity statin users. There were no significant racial differences among the three groups. Statin users tended to have significantly more comorbidities of essential hypertension, diabetes, atrial fibrillation, congestive heart failure, chronic kidney disease, and CAD. Laboratory profiles among 3411 patients with at least one inflammatory marker result within $24 \mathrm{~h}$ of presentation showed that statins users tend to have higher initial oxygen saturation and lower C-reactive protein levels.

Table 2 displays the in-hospital mortality of COVID-19 patients according to statin treatment. A total of 1212 inhospital deaths were noted during 47,673 person-days of follow-up. High-intensity statin users had significantly reduced in-hospital mortality rate (adjusted hazard ratio; aHR 0.53 , 95\% confidential intervals; CI 0.43-0.65) compared to nonstatin users. Low-to-moderate-intensity statin users also had decreased mortality (aHR 0.62, 95\% CI 0.51-0.76). The unadjusted Kaplan-Meier survival curve is shown in Fig. 2. This figure also demonstrated a reduced mortality among statin users.
Fig. 1 Kaplan-Meier curves for hospitalized patients with COVID-19 according to the statin therapy

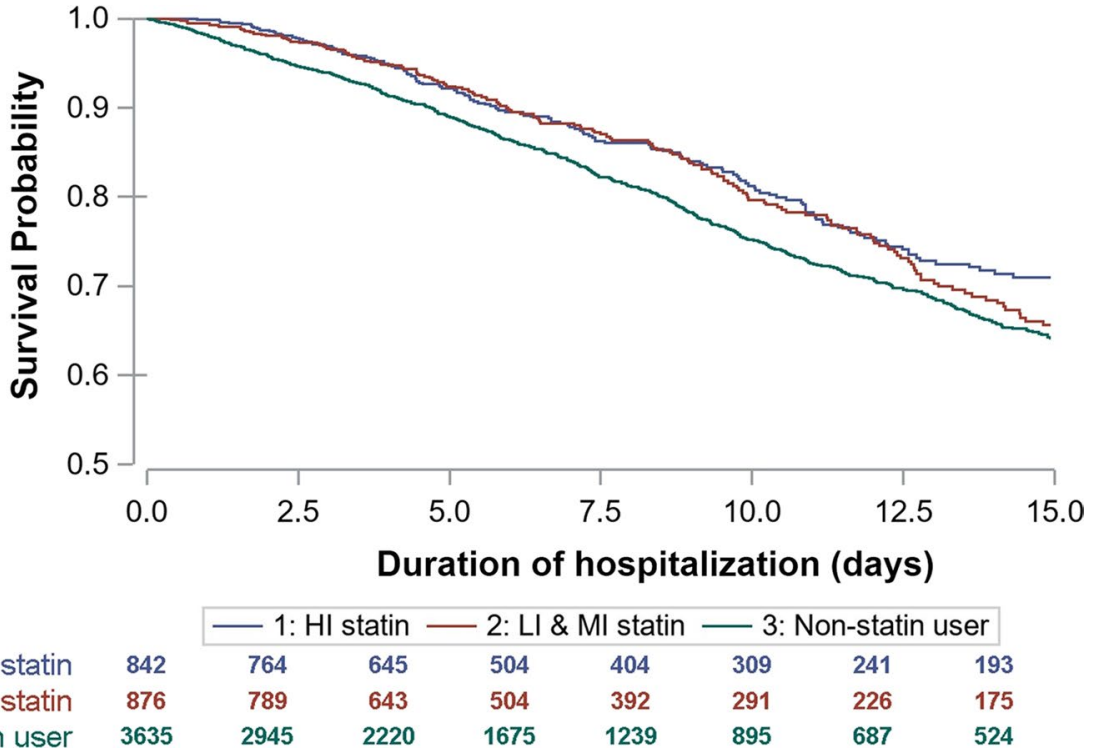

$\begin{array}{rcccccccc}\text { 1: HI statin } & 842 & 764 & 645 & 504 & 404 & 309 & 241 & 193 \\ \text { 2: LI \& Ml statin } & 876 & 789 & 643 & 504 & 392 & 291 & 226 & 175 \\ \text { 3: Non-statin user } & 3635 & 2945 & 2220 & 1675 & 1239 & 895 & 687 & 524\end{array}$




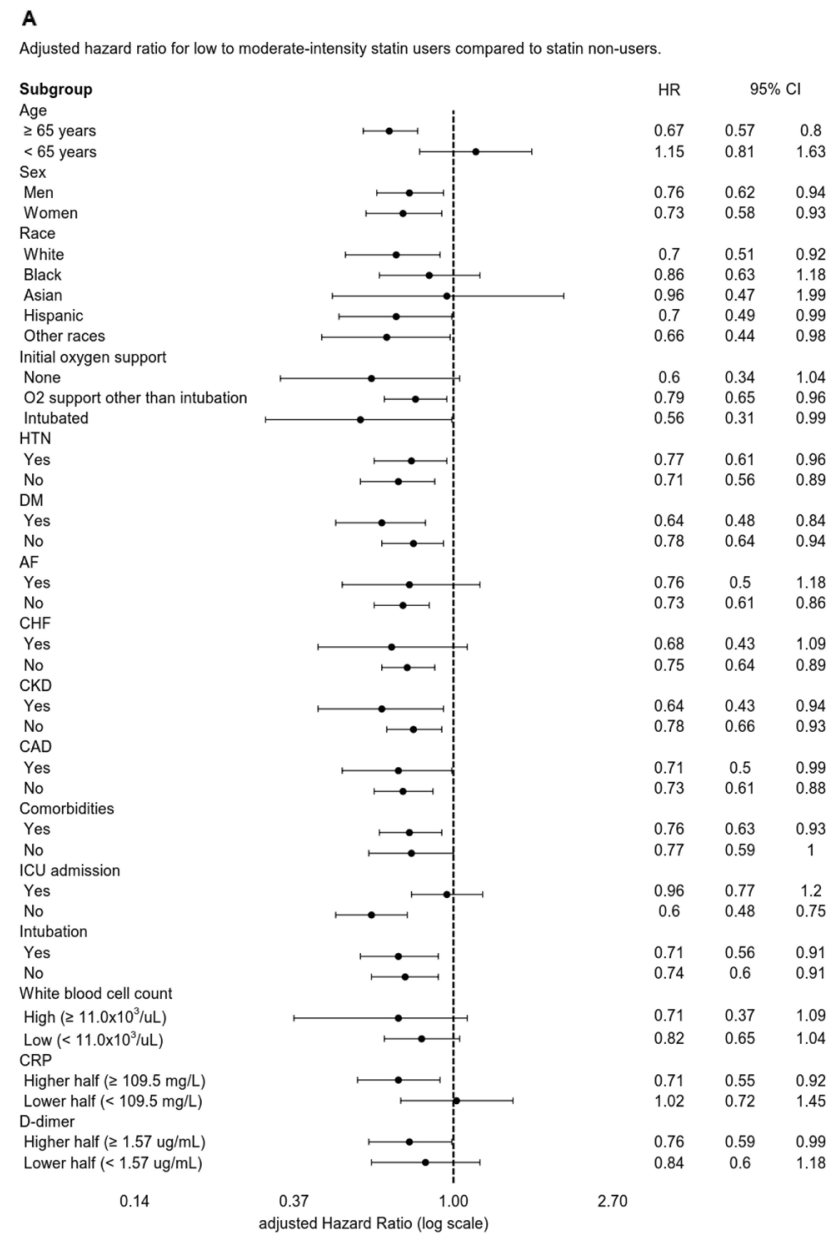

Fig. 2 Stratified analysis on the of statin use and in-hospital mortality in COVID-19 patients according to subgroups of age, sex, race, initial measure of oxygen support, baseline comorbidities (hypertension, diabetes mellitus, atrial fibrillation, congestive heart failure, chronic kidney disease, and CAD), hospital course (intensive care unit admis-

Table 3 shows the result of multivariate Cox proportional regression additionally including initial vital signs and lab data including inflammatory markers. This analysis was performed among subgroup of participants who were tested for initial inflammatory markers within $24 \mathrm{~h}$ of admission. Even after adjustment of initial inflammatory markers, highintensity statin users and low-to-moderate-intensity statin users both had $21 \%$ decreased risk for in-hospital deaths.

The association of statin use and in-hospital mortality according to subgroups of age, sex, race, comorbidities, hospital course, and lab data are depicted in Fig. 2. The use of statin among hospitalized COVID-19 patients was associated with decreased mortality among various subgroups, regardless of age, sex, race, comorbidities, and elevation of inflammatory markers. Particularly, high-intensity statin use was associated with decreased mortality among COVID-19 patients requiring intensive care unit (ICU)
Adjusted hazard ratio for high-intensity statin users compared to statin non-users.

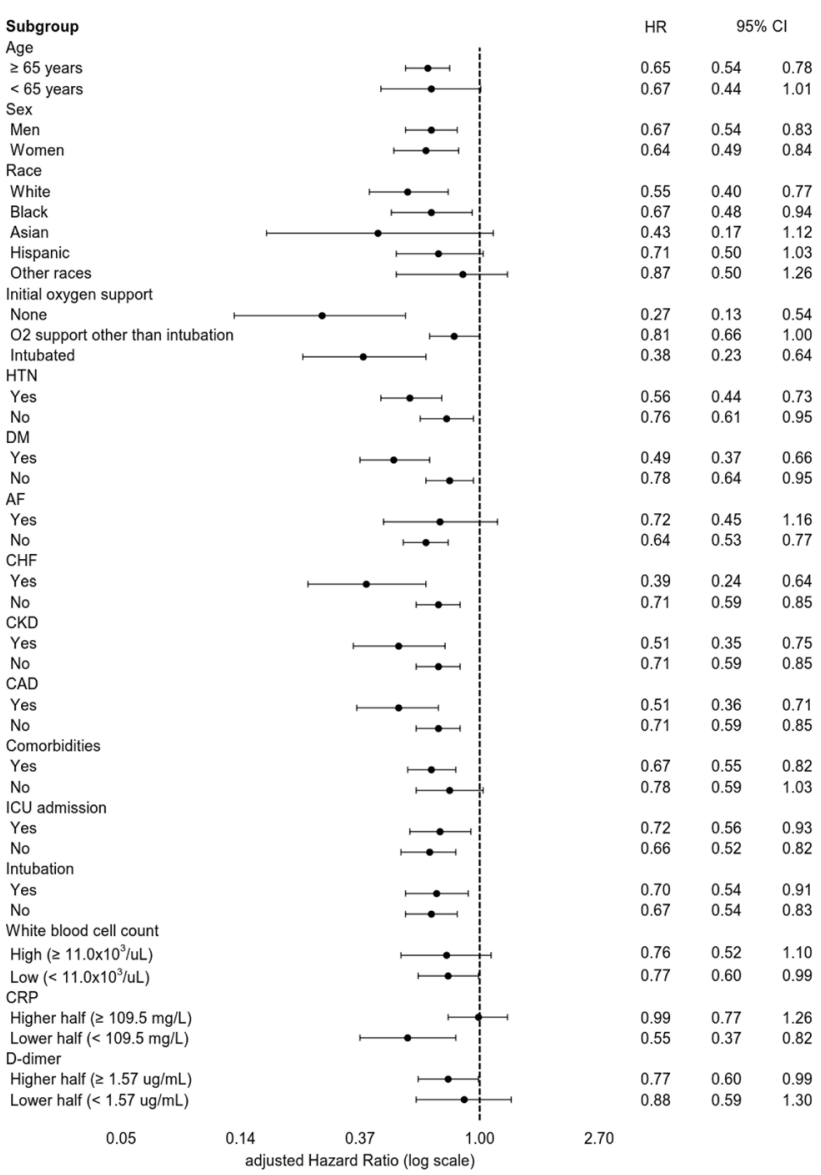

sion, intubation), and baseline laboratory profiles (white blood cell count, C-reactive protein level, D-dimer level). A Adjusted hazard ratio for low-to-moderate-intensity statin users compared to non-statin users. B Adjusted hazard ratio for high-intensity statin users compared to non-statin users

admission (aHR $0.72,95 \%$ CI $0.56-0.93$ ) and intubation (aHR 0.70, 95\% CI 0.54-0.91). The protective effect of high-intensity statin was pronounced among patients with congestive heart failure (aHR 0.39, 95\% CI 0.24-0.64), diabetes (aHR 0.49, 95\% CI 0.37-0.66), chronic kidney disease (aHR 0.51, 95\% CI 0.35-0.75), and for those who required intubation at the initial presentation (aHR 0.38, 95\% CI 0.23-0.64).

The result of sensitivity analysis on incomplete statin users is shown in Table 4. Three hundred thirty-eight out of $1720(19.6 \%)$ statin users were classified as incomplete statin users. These incomplete statin users did not have decreased mortality (aHR 1.04, 95\% CI 0.87-1.23) compared to nonstatin users. Meanwhile, low-to-moderate-intensity statin users (aHR 0.62, 95\% CI 0.51-0.76) and high-intensity statin users (aHR $0.53,95 \%$ CI $0.40-0.64$ ) had significantly reduced mortality compared to non-statin users. 
Table 1 Descriptive characteristics of study population
Table 2 Association of statin use and in-hospital mortality in COVID-19 patients

\begin{tabular}{|c|c|c|c|c|}
\hline & Non-statin user & $\begin{array}{l}\text { Low-to-moderate- } \\
\text { intensity stain user }\end{array}$ & $\begin{array}{l}\text { High-intensity } \\
\text { statin user }\end{array}$ & $p$-value \\
\hline Number of participants (\%) & $3655(68.0)$ & $877(16.3)$ & $843(15.7)$ & \\
\hline \multicolumn{5}{|l|}{$\operatorname{Sex}, N(\%)$} \\
\hline Men & $2030(55.5)$ & $484(55.2)$ & $528(62.6)$ & \multirow[t]{2}{*}{$<0.001$} \\
\hline Women & $1625(44.5)$ & $393(44.8)$ & $315(37.4)$ & \\
\hline Age, mean (SD) & $60.2(18.9)$ & $71.5(12.2)$ & $69.7(12.7)$ & $<0.001$ \\
\hline \multicolumn{5}{|l|}{ Race, $N(\%)$} \\
\hline White & $938(25.7)$ & $242(27.6)$ & $208(24.7)$ & \multirow[t]{5}{*}{0.132} \\
\hline Black & $892(24.4)$ & $214(24.4)$ & $239(28.4)$ & \\
\hline Asian & $148(4.1)$ & $45(5.1)$ & $42(5.0)$ & \\
\hline Hispanic & $1047(28.7)$ & $224(25.5)$ & $220(26.1)$ & \\
\hline Others & $630(17.2)$ & $152(17.3)$ & $134(15.9)$ & \\
\hline \multicolumn{5}{|l|}{ Comorbidity, $N(\%)$} \\
\hline HTN & 973 (26.6) & $454(51.8)$ & $448(53.1)$ & $<0.001$ \\
\hline $\mathrm{DM}$ & $587(16.1)$ & $323(36.8)$ & $337(40.0)$ & $<0.001$ \\
\hline $\mathrm{AF}$ & $182(5.0)$ & $102(11.6)$ & $107(12.7)$ & $<0.001$ \\
\hline $\mathrm{CHF}$ & $164(4.5)$ & $97(11.1)$ & 159 (18.9) & $<0.001$ \\
\hline CKD & $226(7.3)$ & $164(18.7)$ & $186(22.1)$ & $<0.001$ \\
\hline CAD & $249(6.8)$ & $174(19.8)$ & $300(35.6)$ & $<0.001$ \\
\hline \multicolumn{5}{|l|}{ Initial $\mathrm{O} 2$ device } \\
\hline Room air & $1111(30.4)$ & $267(30.4)$ & $267(31.7)$ & \multirow[t]{4}{*}{0.246} \\
\hline NC or NRB or HFNC & $2078(56.9)$ & $526(60.0)$ & $270(55.8)$ & \\
\hline BiPAP or CPAP & $284(7.8)$ & $62(7.1)$ & $66(7.8)$ & \\
\hline Intubated & $182(5.0)$ & $22(2.5)$ & $40(4.7)$ & \\
\hline Participants with lab data & 2278 & 567 & 566 & \\
\hline Temperature, Fahrenheit & $99.9(1.7)$ & 99.7 (1.6) & $99.6(1.6)$ & $<0.001$ \\
\hline $\mathrm{SBP}(\mathrm{mmHg})$ & $140.3(22.4)$ & $144.2(24.5)$ & $149.6(27.1)$ & $<0.001$ \\
\hline $\mathrm{WBC}\left(\times 10^{3} / \mu \mathrm{L}\right)$ & $9.0(5.0)$ & $8.4(4.3)$ & $9.1(6.1)$ & 0.012 \\
\hline $\mathrm{CRP}(\mathrm{mg} / \mathrm{L})$ & $134.1(100.0)$ & 126.7 (99.6) & $110.2(91.3)$ & $<0.001$ \\
\hline D-dimer $(\mu \mathrm{g} / \mathrm{mL})$ & $3.56(4.11)$ & $2.69(3.89)$ & $3.44(4.80)$ & $<0.001$ \\
\hline
\end{tabular}

Acronyms: $N$, number of participants; $S D$, standard deviation; $H T N$, hypertension; $D M$, diabetes mellitus; $A F$, atrial fibrillation; $C H F$, congestive heart failure; $C K D$, chronic kidney disease; $C A D$, coronary artery disease; $N C$, nasal cannula; $N R B$, non-rebreather mask; $H F N C$, high-flow nasal cannula; $B i P A P$, bilevel positive airway pressure; $C P A P$, continuous positive airway pressure; $S B P$, systolic blood pressure; $W B C$, white blood cell count; $C R P, \mathrm{C}$-reactive protein

\begin{tabular}{llll}
\hline & Non-statin user & $\begin{array}{l}\text { Low-to-moderate- inten- } \\
\text { sity stain user }\end{array}$ & High-intensity statin user \\
\hline$N$ & 3655 & 877 & 843 \\
Follow-up period (days) & 30,089 & 8507 & 9077 \\
In-hospital death & 808 & 211 & 193 \\
aHR (95\% CI) & 1.00 (reference) & $0.62(0.51-0.76)$ & $0.53(0.43-0.65)$ \\
\hline
\end{tabular}

Adjusted hazard ratios calculated by Cox proportional hazards regression after adjustments for age; sex; hospital site; race; and history of HTN, DM, AF, CHF, CKD, and CAD

Acronyms: $N$, number of participants; $a H R$, adjusted hazard ratio; $C I$, confidence interval; HTN, hypertension; $D M$, diabetes mellitus; $A F$, atrial fibrillation; $C H F$, congestive heart failure; $C K D$, chronic kidney disease; $C A D$, coronary artery disease 
Table 3 Association of statin use and in-hospital mortality in COVID-19 patients with initial lab data

\begin{tabular}{llll}
\hline & Non-statin user & $\begin{array}{l}\text { Low-to-moderate- inten- } \\
\text { sity stain user }\end{array}$ & High-intensity statin user \\
\hline$N$ & 2278 & 567 & 566 \\
Follow-up period (days) & 21,447 & 5710 & 6275 \\
In-hospital death & 498 & 131 & 129 \\
aHR (95\% CI) & 1.00 (reference) & $0.79(0.64-0.97)$ & $0.79(0.64-0.97)$ \\
\hline
\end{tabular}

Adjusted hazard ratios calculated by Cox proportional hazards regression after adjustments for age; sex; hospital site; race; history of HTN, DM, AF, CHF, CKD, and CAD; initial vital signs (body temperature, systolic blood pressure); and initial lab data (white blood cell count, CRP, and D-dimer)

Acronyms: $N$, number of participants; $a H R$, adjusted hazard ratio; $C I$, confidence interval; $H T N$, hypertension; $D M$, diabetes mellitus; $A F$, atrial fibrillation; $C H F$, congestive heart failure; $C K D$, chronic kidney disease; $C A D$, coronary artery disease; $C R P, \mathrm{C}$-reactive protein

Table 4 Sensitivity analysis for association of statin use and in-hospital mortality in COVID-19 patients considering in-hospital statin compliance

\begin{tabular}{lllll}
\hline & Non-statin user & Incomplete statin user & $\begin{array}{l}\text { Low-to-moderate- inten- } \\
\text { sity stain user }\end{array}$ & High-intensity statin user \\
\hline$N$ & 3655 & 338 & 707 & 675 \\
Follow-up period (days) & 30,089 & 5053 & 6067 & 6463 \\
In-hospital death & 808 & 168 & 126 & 110 \\
aHR (95\% CI) & 1.00 (reference) & $1.04(0.87-1.23)$ & $0.62(0.51-0.76)$ & $0.53(0.43-0.65)$ \\
\hline
\end{tabular}

Adjusted hazard ratios calculated by Cox proportional hazards regression after adjustments for age; sex; hospital site; race; and history of HTN, $\mathrm{DM}, \mathrm{AF}, \mathrm{CHF}, \mathrm{CKD}$, and CAD

Acronyms: $N$, number of participants; $a H R$, adjusted hazard ratio; $C I$, confidence interval; $H T N$, hypertension; $D M$, diabetes mellitus; $A F$, atrial fibrillation; $C H F$, congestive heart failure; $C K D$, chronic kidney disease; $C A D$, coronary artery disease

The propensity score matching yielded 1445 matched controls. The characteristic of statin users and matched controls are shown in Supplementary Table 1. As all standardized differences were less than 0.10 , the matched cohort was considered well balanced. Statin users had reduced in-hospital mortality (aHR $0.72,95 \%$ CI $0.62-0.83$ ) compared to propensity score-matched controls (Supplementary Table 2). Lastly, in falsification endpoint analysis, both low-to-moderate-intensity statin use (aHR 0.90, 95\% CI 0.61-1.29) and high-intensity statin use (aHR $1.21,95 \%$ CI $0.86-1.73$ ) were not associated with severe anemia requiring blood transfusion, which implies that the reduced mortality upon statin use on main analysis is unlikely driven by hidden bias.

\section{Discussion}

In this multi-hospital retrospective cohort study of 5375 hospitalized COVID-19 patients, we demonstrated that inhospital use of statin was associated with reduced mortality. Multiple comorbidities, potential confounders, and laboratory data of inflammatory markers that were not considered in the prior studies were implemented in this study. The protective effect of statin among hospitalized COVID-19 patients was consistent among various subgroups of age, sex, race, and comorbidities. The dose of statins therapy was also considered and high-intensity statin was associated with significantly decreased mortality rates among severe COVID-19 patients requiring ICU admission or intubation. The protective effect of statin tended to be prominent among participants who had elevated D-dimer at the time of presentation.

Previous studies on hospitalized COVID-19 patients have reported that the antecedent use of statin was associated with lower levels of inflammatory markers at the time of presentation and decreased in-hospital mortality [16-19, 30]. However, studies of the in-hospital use of statin among COVID-19 patients have been inconsistent. While a few observational studies have reported favorable outcomes of statin users especially on high-risk subgroups $[20,21,24]$, they had limitations such as small sample size, lack of potential confounders, and limited generalizability. Because some studies report a null association of statin use and mortality [23, 31-33] while others with pooled analysis still suggest the benefit of statin [22, 33], there is no consensus on statin therapy among hospitalized COVID-19 patients. Our study aimed to address the limitations of previous studies and have demonstrated the benefits of statin use among a large number of hospitalized 
COVID-19 patients after adjusting for various potential confounders among multiple subgroups, including racial diversity.

The potential beneficial effects of statin among COVID19 patients have been proposed based on several mechanisms. First, underlying cardiovascular disease or comorbidity is a major risk factor of severe COVID-19 and a predictor of poor prognosis [5, 34, 35]. Therefore, statin has been suggested for possible benefits among COVID-19 patients by providing cardioprotective effects and preventing adverse cardiac events. Second, statin has various pleiotropic effects beyond its cholesterol-lowering properties. These pleiotropic effects include immune modulation in various levels, decrease in oxidative stress, antithrombotic effect, and improvement of endothelial function $[9,36]$. Also, some studies have found that statins can mitigate the hyperactive innate immune response in COVID-19. Prior studies have found that SARS-CoV can induce the myeloid differentiation primary response 88 (MyD88) pathway [37], which is a key driver of Toll-like receptor (TLR) responses and has been associated with excessive inflammation. Because statins are known to suppress the MyD88 pathway, the beneficial associations we observed could be related to a tuning of the overactive MyD88 response seen in COVID-19 [37, 38]. Lastly, prior studies that evaluated statin also suggested a preventive effect on acute respiratory distress syndrome and reported that statin use was associated with decreased mortality, especially on viral illnesses [39-41].

Few interesting associations were noted in the subgroup analysis of our studies. High-intensity statin had an even more protective effect compared to low-to-moderate-intensity statin among high-risk COVID-19 subgroups, such as patients with hypertension, diabetes, congestive heart failure, $\mathrm{CAD}$, and those requiring ICU admission. Also, while most other subgroups did not show a significant difference between low-to-moderate-intensity statin and high-intensity statin use, certain subgroups (patients with ages less than 65 years and Black race) only had significant benefits from the use of high-intensity statin. Further studies on the association of racial difference with the severity of COVID-19 would be needed.

Subgroup analysis based on C-reactive protein level is also notable since those presented with higher levels had a favorable outcome with low-to-moderate-intensity statin. Meanwhile, those who presented with lower C-reactive protein levels had significantly reduced mortality with highintensity statin use. This finding might be related to antecedent statin use prior to admission, as statin users had a lower level of C-reactive protein levels upon presentation [17]. Participants presented with higher C-reactive protein with antecedent high-intensity statin use are more likely to have severe COVID-19 infection that would result in high mortality.
There are some limitations to consider when interpreting the result of our study. First, this is an observational retrospective study that has the inherent limitation of a possible reverse-causality. Prospective randomized trials would be needed to confirm our findings. Second, there were participants who were still hospitalized at the time of data freeze and censored prior to completion of hospitalization course. However, these incomplete data were from 101 out of a total 5375 participants comprising less than $2 \%$ of the study population. Third, lipid profiles and statin use prior to admission were not considered. However, lipid profiles tend to be altered in the acute setting of infection and would provide less reliable and potentially spurious information for risk stratification [42]. Nonetheless, as benefits of antecedent stain use were reported among COVID-19 patients [16-19], future studies among COVID-19 patients without previous history of statin prescription would be merited. Lastly, severity of disease and treatments received were not considered. However, we have tried to take these into account and added initial measure of oxygen support as a major covariate, which would reflect the severity of disease.

Despite limitations, our study has notable strengths. We took account of multiple comorbidities and confounders that were not considered in the previous studies. Also, the study was performed among a large number of hospitalized COVID-19 patients with racial diversity. Third, the dose of statin was also considered in the analysis. Fourth, laboratory data including major inflammatory markers (white blood cell count, C-reactive protein, and D-dimer) were considered in our analysis. Finally, the result was consistent among multiple subgroup analyses; sensitivity analysis, propensity score-matched cohort analysis, and also falsification analysis extend the generalizability and reliability of our findings.

In conclusion, statin use among hospitalized COVID-19 patients was associated with reduced in-hospital mortality even after adjustment of multiple potential confounding variables and inflammatory markers. The results were also consistent among various subgroup populations. These results suggest statin therapy may be helpful among hospitalized COVID-19 patients.

Supplementary Information The online version contains supplementary material available at https://doi.org/10.1007/s10557-021-07263-2.

Author Contribution Drs. Choi and Rosenson had full access to all of the data in the study and take responsibility for the integrity of the data and the accuracy of the data analysis.

Concept and design: All authors.

Acquisition, analysis, or interpretation of data: Choi, Chen, Goonewardena, Rosenson.

Drafting of the manuscript: Choi, Goonewardena, Rosenson.

Critical revision of the manuscript for important intellectual content: All authors.

Statistical analysis: Choi, Chen, Rosenson.

Obtained funding: Rosenson. 
Administrative, technical, or material support: Chen, Pacheco, Mejia, Smith.

Supervision: Goonewardena, Rosenson.

Funding The CURA Foundation provided support for data retrieval from electronic medical records.

Availability of Data and Material (Data Transparency) Formal requests to access the dataset need to be sent to the COVID-19 Committee, Icahn School of Medicine.

Code Availability The codes involved in this study are available from the corresponding author upon reasonable request.

\section{Declarations}

Ethics Approval The Mount Sinai Institutional Review Board approved this study (IRB number: 20-03558).

Consent to Participate Not applicable.

Consent for Publication Not applicable.

Conflict of Interest The authors declare no competing interests.

Disclaimer The study sponsors had no role in the design and conduct of the study; collection, management, analysis, and interpretation of the data; preparation, review, or approval of the manuscript; or decision to submit the manuscript for publication.

\section{References}

1. World Health Organization. WHO coronavirus disease (COVID19) dashboard. https://covid19.who.int/. Accessed 14 Sept 2021.

2. Pinney SP, Giustino G, Halperin JL, Mechanick JI, Neibart E, Olin $\mathrm{JW}$, et al. Coronavirus historical perspective, disease mechanisms, and clinical outcomes: JACC focus seminar. J Am Coll Cardiol. 2020;76:1999-2010.

3. Giustino G, Pinney SP, Lala A, Reddy VY, Johnston-Cox HA, Mechanick JI, et al. Coronavirus and cardiovascular disease, myocardial injury, and arrhythmia: JACC focus seminar. J Am Coll Cardiol. 2020;76:2011-23.

4. Bhatraju PK, Ghassemieh BJ, Nichols M, Kim R, Jerome KR, Nalla AK, et al. COVID-19 in critically ill patients in the Seattle region - case series. N Engl J Med. 2020;382:2012-22.

5. Shi S, Qin M, Shen B, Cai Y, Liu T, Yang F, et al. Association of cardiac injury with mortality in hospitalized patients with COVID-19 in Wuhan, China. JAMA Cardiol. 2020;5:802-10.

6. Colantonio LD, Shannon ED, Orroth KK, Zaha R, Jackson EA, Rosenson RS, Exter J, Mues KE, Muntner P. Ischemic event rates in very-high-risk adults. J Am Coll Cardiol. 2019;74:2496-507.

7. Bikdeli B, Madhavan MV, Gupta A, Jimenez D, Burton JR, Der Nigoghossian C, et al. Pharmacological agents targeting thromboinflammation in COVID-19: review and implications for future research. Thromb Haemost. 2020;120:1004-24.

8. Ridker PM, Danielson E, Fonseca FA, Genest J, Gotto AM Jr, Kastelein JJ, et al. Rosuvastatin to prevent vascular events in men and women with elevated C-reactive protein. N Engl J Med. 2008;359:2195-207.

9. Oesterle A, Laufs U, Liao JK. Pleiotropic effects of statins on the cardiovascular system. Circ Res. 2017;120:229-43.
10. Kong F, Ye B, Lin L, Cai X, Huang W, Huang Z. Atorvastatin suppresses NLRP3 inflammasome activation via TLR4/MyD88/NFkappab signaling in PMA-stimulated THP-1 monocytes. Biomed Pharmacother. 2016;82:167-72.

11. Hoffmann M, Kleine-Weber H, Schroeder S, Kruger N, Herrler T, Erichsen S, et al. Sars-CoV-2 cell entry depends on ACE2 and TMPRSS 2 and is blocked by a clinically proven protease inhibitor. Cell. 2020;181:271-280 e278.

12. Shin YH, Min JJ, Lee JH, Kim EH, Kim GE, Kim MH, et al. The effect of fluvastatin on cardiac fibrosis and angiotensin-converting enzyme-2 expression in glucose-controlled diabetic rat hearts. Heart Vessels. 2017;32:618-27.

13. Tikoo K, Patel G, Kumar S, Karpe PA, Sanghavi M, Malek V, et al. Tissue specific up regulation of ACE2 in rabbit model of atherosclerosis by atorvastatin: role of epigenetic histone modifications. Biochem Pharmacol. 2015;93:343-51.

14. Li W, Moore MJ, Vasilieva N, Sui J, Wong SK, Berne MA, et al. Angiotensin-converting enzyme 2 is a functional receptor for the sars coronavirus. Nature. 2003;426:450-4.

15. Rosenson RS, Baker S, Banach M, Borow KM, Braun LT, Bruckert E, et al. Optimizing cholesterol treatment in patients with muscle complaints. J Am Coll Cardiol. 2017;70:1290-301.

16. Daniels LB, Sitapati AM, Zhang J, Zou J, Bui QM, Ren J, et al. Relation of statin use prior to admission to severity and recovery among COVID-19 inpatients. Am J Cardiol. 2020;136:149-55.

17. Gupta A, Madhavan MV, Poterucha TJ, DeFilippis EM, Hennessey JA, Redfors B, et al. Association between antecedent statin use and decreased mortality in hospitalized patients with COVID-19. Nat Commun. 2021;12:1325.

18. Lohia P, Kapur S, Benjaram S, Mir T. Association between antecedent statin use and severe disease outcomes in COVID-19: a retrospective study with propensity score matching. J Clin Lipidol. 2021;15:451-9.

19. Daniels LB, Ren J, Kumar K, Bui QM, Zhang J, Zhang X, et al. Relation of prior statin and anti-hypertensive use to severity of disease among patients hospitalized with COVID-19: findings from the American Heart Association's COVID-19 cardiovascular disease registry. PLoS One. 2021;16:e254635.

20. Saeed O, Castagna F, Agalliu I, Xue X, Patel SR, Rochlani Y, et al. Statin use and in-hospital mortality in patients with diabetes mellitus and covid-19. J Am Heart Assoc. 2020;9:e018475.

21. Zhang XJ, Qin JJ, Cheng X, Shen L, Zhao YC, Yuan Y, et al. Inhospital use of statins is associated with a reduced risk of mortality among individuals with covid-19. Cell Metab. 2020;32:176187 e174.

22. Kow CS, Hasan SS. Meta-analysis of effect of statins in patients with covid-19. Am J Cardiol. 2020;134:153-5.

23. Butt JH, Gerds TA, Schou M, Kragholm K, Phelps M, HaversBorgersen E, et al. Association between statin use and outcomes in patients with coronavirus disease 2019 (covid-19): a nationwide cohort study. BMJ Open. 2020;10:e044421.

24. De Spiegeleer A, Bronselaer A, Teo JT, Byttebier G, De Tre G, Belmans L, et al. The effects of ARBs, ACEis, and statins on clinical outcomes of COVID-19 infection among nursing home residents. J Am Med Dir Assoc. 2020;21:909-914 e902.

25. Choi D, Choi S, Kim H, Kim K, Kim N, Ko A, et al. Impact of continuity of care on cardiovascular disease risk among newlydiagnosed hypertension patients. Sci Rep. 2020;10:19991.

26. Kozma CM, Dickson M, Phillips AL, Meletiche DM. Medication possession ratio: Implications of using fixed and variable observation periods in assessing adherence with disease-modifying drugs in patients with multiple sclerosis. Patient Prefer Adherence. 2013;7:509-16.

27. Sikka R, Xia F, Aubert RE. Estimating medication persistency using administrative claims data. Am J Manag Care. 2005;11:449-57. 
28. Prasad V, Jena AB. Prespecified falsification end points: can they validate true observational associations? JAMA. 2013;309:241-2.

29. Wimmer NJ, Resnic FS, Mauri L, Matheny ME, Yeh RW. Comparison of transradial versus transfemoral percutaneous coronary intervention in routine practice: evidence for the importance of "falsification hypotheses" in observational studies of comparative effectiveness. J Am Coll Cardiol. 2013;62:2147-8.

30. Oh TK, Song I, Jeon YT. Statin therapy and the risk of COVID19: a cohort study of the national health insurance service in South Korea. J Pers Med. 2021;11(2):116.

31. Hariyanto TI, Kurniawan A. Statin therapy did not improve the in-hospital outcome of coronavirus disease 2019 (COVID-19) infection. Diabetes Metab Syndr. 2020;14:1613-5.

32. Hariyanto TI, Kurniawan A. Statin and outcomes of coronavirus disease 2019 (covid-19): a systematic review, meta-analysis, and meta-regression. Nutr Metab Cardiovasc Dis. 2021;31:1662-70.

33. Pal R, Banerjee M, Yadav U, Bhattacharjee S. Statin use and clinical outcomes in patients with COVID-19: an updated systematic review and meta-analysis. Postgrad Med J. 2021. postgradmedj-2020-139172.

34. Yang J, Zheng Y, Gou X, Pu K, Chen Z, Guo Q, et al. Prevalence of comorbidities and its effects in patients infected with SARSCoV-2: a systematic review and meta-analysis. Int J Infect Dis. 2020;94:91-5.

35. Zhou F, Yu T, Du R, Fan G, Liu Y, Liu Z, et al. Clinical course and risk factors for mortality of adult inpatients with COVID19 in Wuhan, China: a retrospective cohort study. Lancet. 2020;395:1054-62.

36. Zeiser R. Immune modulatory effects of statins. Immunology. 2018;154:69-75.
37. Yuan S. Statins may decrease the fatality rate of middle east respiratory syndrome infection. mBio. 2015;6:e01120.

38. Totura AL, Whitmore A, Agnihothram S, Schafer A, Katze MG, Heise MT, et al. Toll-like receptor 3 signaling via TRIF contributes to a protective innate immune response to severe acute respiratory syndrome coronavirus infection. mBio. 2015;6:e00638-00615.

39. Frost FJ, Petersen H, Tollestrup K, Skipper B. Influenza and COPD mortality protection as pleiotropic, dose-dependent effects of statins. Chest. 2007;131:1006-12.

40. McAuley DF, Laffey JG, O'Kane CM, Perkins GD, Mullan B, Trinder TJ, et al. Simvastatin in the acute respiratory distress syndrome. N Engl J Med. 2014;371:1695-703.

41. Vandermeer ML, Thomas AR, Kamimoto L, Reingold A, Gershman K, Meek J, et al. Association between use of statins and mortality among patients hospitalized with laboratory-confirmed influenza virus infections: a multistate study. J Infect Dis. 2012;205:13-9.

42. Roccaforte V, Daves M, Lippi G, Spreafico M, Bonato C. Altered lipid profile in patients with COVID-19 infection. J Lab Precis Med. 2021;6:2-2.

Publisher's Note Springer Nature remains neutral with regard to jurisdictional claims in published maps and institutional affiliations. 